\title{
HD 203030B: AN UNUSUALLY COOL YOUNG SUBSTELLAR COMPANION NEAR THE L/T TRANSITION
}

\author{
Stanimir A. Metchev \\ Department of Physics and Astronomy, UCLA, Los Angeles, CA 90095; metchev@astro.ucla.edu \\ AND \\ Lynne A. Hillenbrand \\ Division of Physics, Mathematics, and Astronomy, California Institute of Technology, Pasadena, CA 91125; 1ah@astro.caltech.edu \\ Received 2006 April 21; accepted 2006 July 20
}

\begin{abstract}
We present the discovery of a brown dwarf companion to the star HD 203030: a G8 V Solar analog with an estimated age between 130 and 400 Myr old G8 V. Separated by $11^{\prime \prime} 9$ (487 AU in projection) from its host star, HD 203030B has an estimated mass of $0.023_{-0.011}^{+0.008} M_{\odot}$. The $K$-band spectral type of L7.5 \pm 0.5 places HD 203030B near the critical $\mathrm{L} / \mathrm{T}$ transition in brown dwarfs, which is characterized by the rapid disappearance of dust in substellar photospheres. From a comparative analysis with well-characterized field L/T transition dwarfs, we find that, despite its young age, HD 203030B has a bolometric luminosity similar to the $>1$ Gyr old field dwarfs. Adopting a radius from current models of substellar evolution, we hence obtain that the effective temperature of HD 203030B is only $1206_{-116}^{+74} \mathrm{~K}$, markedly lower than the $\approx 1440 \mathrm{~K}$ effective temperatures of field $\mathrm{L} / \mathrm{T}$ transition dwarfs. The temperature discrepancy can be resolved if either (1) the ages of field brown dwarfs have been overestimated by a factor of $\approx 1.5$, leading to underestimated radii, or (2) the lower effective temperature of HD 203030B is related to its young age, implying that the effective temperature at the $\mathrm{L} / \mathrm{T}$ transition is gravity dependent.
\end{abstract}

Subject headings: binaries: general — instrumentation: adaptive optics — stars: individual (HD 203030) stars: low-mass, brown dwarfs

Online material: color figures

\section{INTRODUCTION}

After more than a decade of study, the fundamental parameters of brown dwarfs remain poorly constrained. Unlike hydrogenburning stars, whose effective temperatures and luminosities are approximately age independent (at a fixed mass and composition) on the main sequence, brown dwarfs cool and dim continuously as they age. Only one of the fundamental substellar parameters, luminosity, can be estimated independently of the others, through the use of trigonometric parallax and empirically determined bolometric corrections. Any attempts to resolve the remaining degeneracies among temperatures, ages, masses, and radii rely on the fortuitous discovery of brown dwarfs in association with stars or clusters of known age. In such cases brown dwarf ages can be inferred by demonstrating the physical association of the brown dwarfs, usually as common proper motion secondaries or as members of stellar associations, with stars that have known ages. Brown dwarf masses can be measured from orbital astrometry and radial velocity monitoring of close $(<5-10 \mathrm{AU})$ binary systems, in which at least one component is substellar. Finally, brown dwarf radii, and hence effective temperatures and surface gravities, can be measured in substellar eclipsing binaries. The union of these three fortuitous cases, eclipsing brown dwarf binaries associated with stars, offers the best opportunity for empirical determination of substellar parameters. However, such unusual systems are extremely rare - the first one, 2MASS J05352184-0546085, has only recently been reported (Stassun et al. 2006). In all other cases involving brown dwarfs, the estimation of their properties relies to various degrees on the use of theoretical models of substellar evolution (e.g., Burrows et al. 1997, 2001; Chabrier et al. 2000; Baraffe et al. 2003).

Consequently, substellar evolution models remain for the most part empirically unconfirmed. Partial tests have been carried out for systems other than the ideal eclipsing double-line systems. Close et al. (2005) and Luhman et al. (2005) used astrometric measurements of the multiple system $\mathrm{AB}$ Dor $\mathrm{A} / \mathrm{B} / \mathrm{C}$ to test the mass-age-luminosity relation at the stellar-substellar boundary. The two analyses offer examples of how substellar evolution models can be tested without considering all degenerate substellar parameters - in this case excluding effective temperature. We note, however, that the conclusions of the two teams differ (see Nielsen et al. 2005 and Luhman \& Potter 2006 for further discussion), in part because the stellar parameters themselves (in particular the stellar ages), although used as a reference in both studies, may often not be known to the desired or believed level of accuracy. In a separate example, Mohanty et al. (2004a, 2004b) tackle the full set of degenerate substellar parameters using highresolution spectroscopic observations of brown dwarfs in the Upper Scorpius and Taurus associations. Their studies rely on model spectra of brown dwarf photospheres, constructed independently of substellar evolutionary models, to resolve the mass-effective temperature-luminosity degeneracy. Even though model dependent, they allow a self-consistent comparison between substellar cooling and photospheric models in the context of empirical data.

In the present paper we take a similar approach and discuss the degeneracies among substellar age, luminosity, and effective temperature (i.e., excluding mass) in the context of models of substellar evolution. We address these fundamental properties with the help of a new brown dwarf that we discovered as a common proper motion companion to HD 203030 (HIP 105232; $\S 2$ ), a 130-400 Myr old G8 V star. The companion, HD 203030B, has a spectral type of L7.5, and thus lies very near the transition between L- and T-type substellar photospheres, characterized by the settling of dust and the appearance of methane absorption in the near-IR spectra of brown dwarfs. Because dust settling occurs over a very narrow range of effective temperatures, 1500 $1300 \mathrm{~K}$ (spectral types L6-T4), a fact inferred both theoretically 
TABLE 1

OBSERVATIONS OF HD 203030

\begin{tabular}{|c|c|c|c|}
\hline Epoch (UT) & Photometric? & Observing Mode & Telescope \\
\hline 2002 Aug $28 \ldots .$. & Yes & $J H K_{S}$ imaging & Palomar \\
\hline 2003 Jul $16 \ldots \ldots$. & No & $K_{S}$ imaging & Palomar \\
\hline 2004 Jun $26 \ldots \ldots$ & No & $K_{S}$ imaging & Palomar \\
\hline $2005 \mathrm{Jul} 14 \ldots \ldots .$. & Yes & $J K_{S}$ imaging, $K$ spectroscopy & Keck \\
\hline
\end{tabular}

(Ackerman \& Marley 2001; Tsuji 2002) and semi-empirically (Golimowski et al. 2004; Vrba et al. 2004), the effective temperature of HD 203030B is expected to be constrained very well. Given the known age and luminosity of HD 203030B, we can find its radius and thus offer a constraint on the theory $(\S 3)$. Our approach is not fully empirical, because it relies on the effective temperature scales of Golimowski et al. and Vrba et al., both of which rely on evolution models for the substellar age-mass-radius relations. However, by adopting the same theoretical evolution models (Burrows et al. 1997, 2001) as in these two studies, our analysis provides a test of the self-consistency of the theoretical models $(\S 4)$

\section{OBSERVATIONS: DETECTION AND FOLLOW-UP OF HD 203030B}

HD 203030B was discovered in the course of a direct imaging AO survey for substellar companions conducted at the Palomar Observatory with the Hale 200 inch $(5 \mathrm{~m})$ and the Keck II $10 \mathrm{~m}$ telescope. The observation strategy for the survey is described in Metchev \& Hillenbrand (2004) and Metchev (2006). Here we briefly describe aspects relevant to HD 203030.

\subsection{Imaging: Detection of HD 203030B}

HD 203030B was first detected at the Palomar $5 \mathrm{~m}$ telescope, using the facility AO system PALAO (Troy et al. 2000) and the Palomar High-Angular Resolution Observer (PHARO; Hayward et al. 2001) camera. PALAO allows diffraction-limited (0".09 at $K_{S}$ band) imaging in the near-IR with $K_{S}$-band Strehl ratios routinely above $50 \%$ for natural guide stars brighter than $R=10$ mag. PHARO employs a $1024 \times 1024$ pixel $\mathrm{HgCdTe}$ HAWAII detector, allowing a $25^{\prime \prime} .7 \times 25^{\prime \prime} .7$ field of view $($ FOV $)$ at a pixel scale of 25.09 mas pixel $^{-1}$. We used a 0.97 diameter opaque coronagraphic spot in PHARO to occult the primary in deep (60 s) exposures.

HD 203030 was imaged at Palomar on three occasions: 2002 August 28, 2003 July 16, and 2004 June 26 (Table 1). During the first (discovery) epoch, we obtained a total of 24 individual $60 \mathrm{~s} \mathrm{ex}$ posures of the primary with the coronagraph. Subsequent epoch imaging was shorter (6-12 minutes), as dictated by the necessity of obtaining only astrometric follow-up of the already discovered candidate companions. In addition to the deep coronagraphic exposures, at each of the three epochs we obtained shallow (2-10 s) noncoronagraphic $K_{S}$-band exposures of HD 203030 in a fivepoint dither pattern to use as references for photometry and astrometry. We also took five $2 \mathrm{~s} J$ - and $H$-band unocculted exposures at first epoch to measure the colors of the brightest candidate companions. A $1 \%$ neutral density (ND) filter was placed in the optical path in PHARO during the second and the third imaging epochs to prevent the primary from saturating in the unocculted $K_{S}$-band exposures. The short $J H K_{S}$ exposures from the first epoch, however, were unattenuated, and the primary was saturated.

A final (fourth) set of $J K_{S}$ imaging observations was obtained with the Keck II adaptive optics (AO) system (Wizinowich et al.

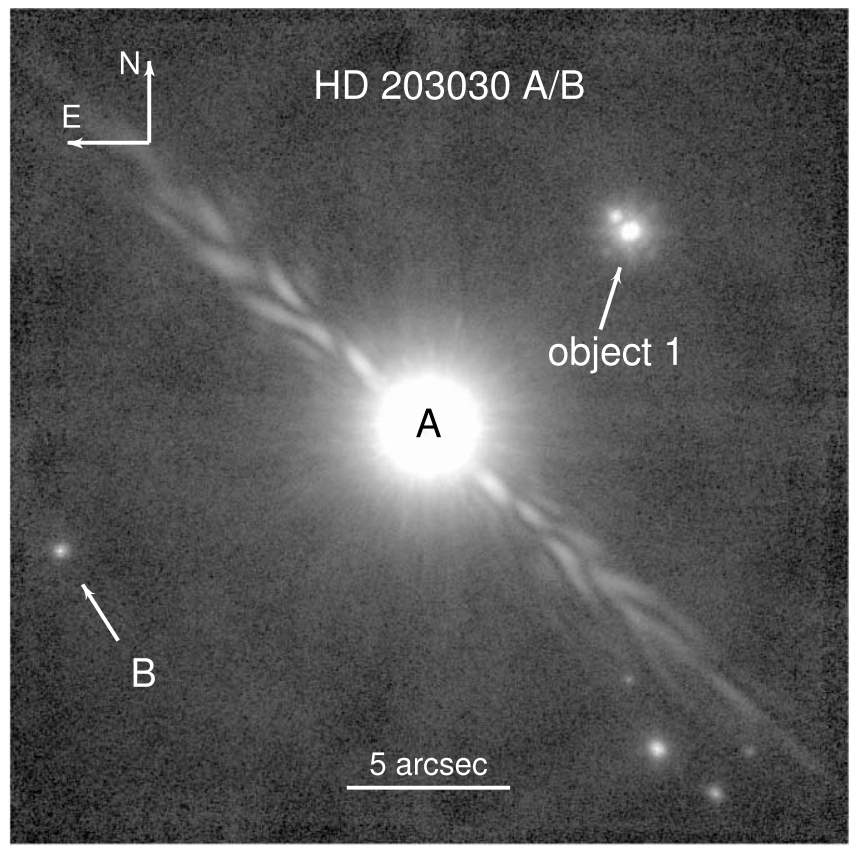

FIG. 1.-Discovery image of HD 203030B, obtained at Palomar. The total exposure time is 24 minutes at $K_{S}$ band. The primary, HD 203030A, is occulted by a 0.97 coronagraphic spot. The secondary, HD 203030B, is visible $11^{\prime \prime} .9$ (487 AU) away near the eastern edge of the image. Six other background stars are also visible in the $25^{\prime \prime} .7 \times 25^{\prime \prime} .7$ field of view. Object 1 was used for bootstrapping photometric and astrometric measurements. The wavy feature running diagonally across the image is an artifact due to a temporary oil streak on the secondary mirror of the telescope, present only in our 2002 data.

2000) on 2005 July 12. On Keck we used the near-IR camera NIRC2 (K. Matthews et al. 2006, in preparation) in its 20 mas pixel $^{-1}$ scale and with a 1.0 diameter partially transmissive coronagraph. Both AO systems were employed in natural guidestar mode, using HD $203030(V=8.4 \mathrm{mag})$ itself as the guide star, which allowed $K_{S}$-band Strehl ratios around $50 \%$ for all observations.

The reduction of the imaging data followed the standard steps of sky subtraction, flat fielding, and bad-pixel correction, as described in detail in Metchev \& Hillenbrand (2004). The individual processed images were median-combined to produce a final high signal-to-noise ratio image. The final first-epoch image of HD 203030 is shown in Figure 1. A total of seven candidate companions are visible in the PHARO FOV, only one of which, HD 203030B (indicated with an arrow near the eastern edge of the image), was confirmed as a bona fide proper-motion companion. The brightest field object ("object 1" in Fig. 1) was a background star that was visible also in the short exposures and was used to bootstrap the relative photometry and astrometry of HD 203030B.

\subsubsection{Astrometry: Confirmation of Common Proper Motion}

Measurements of the relative position of HD 203030B with respect to that of HD 203030A were obtained at all four imaging epochs. Whenever possible, relative astrometry was obtained from images showing both the primary and the companion: the unocculted Palomar images from the first epoch, in which the primary was saturated, and the occulted Keck images from the last epoch, during which we used the partially transmissive NIRC2 coronagraph. By fitting Gaussians to the PSFs of HD 203030A (or to its wings, if saturated) and of HD 203030B, we could measure each of their positions with $2-5$ mas (0.04-0.10 pixels) precision. This was not possible for the images taken during the 

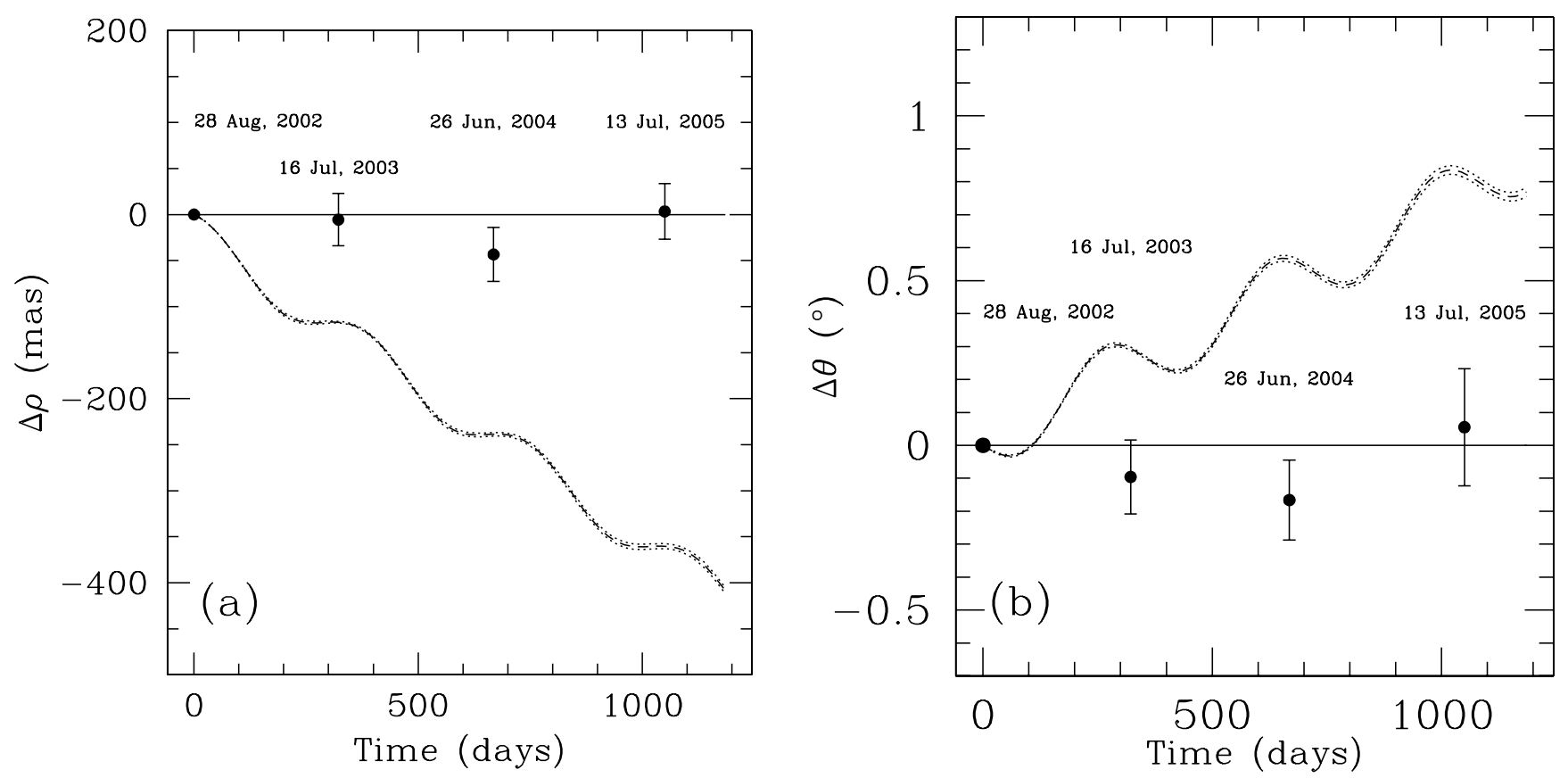

FIG. 2.- Relative proper-motion diagrams for HD 203030B, showing the change in radial separation $(a)$ and position angle $(b)$ with respect to the position of HD $203030 \mathrm{~A}$. The astrometric measurements at the four observational epochs are denoted with dots. The errors at the first epoch are not shown separately but are absorbed into the error bars of the measurements at the subsequent epochs. The solid line in both panels traces null relative proper motion, i.e., common proper motion. The dashed and dotted lines in the panels trace the expected relative motion of $\mathrm{HD} 203030 \mathrm{~B}$ and its $1 \sigma$ error if the companion were an unrelated background star with negligible apparent proper motion.

second and third Palomar epochs. Because we used the PHARO ND filter for the short exposures at these epochs, the companion was not visible in the exposures in which the primary was unocculted. And because the PHARO coronagraph is opaque, the primary was obscured in the deep coronagraphic exposures, in which the secondary was visible. In the latter cases we estimated the position of the occulted primary by either (1) bootstrapping its position in the coronagraphic images from the position of object 1 (Fig. 1) visible in the shallow exposures taken at the same epoch or (2) inferring its position from the peak of the twodimensional cross-correlation of the central $512 \times 512$ pixel section of the coronagraphic image with a copy of itself inverted around its central pixel. The latter technique takes advantage of the fact that the extended PSF halo of the primary is the dominant source of signal in the image and that the halo is approximately radially symmetric on angular scales larger than the radius of the coronagraph. Using one or the other of these two approaches, we constrained the position of the primary behind the coronagraph to within 13 mas $(\approx 0.5$ pixels $)$.

At this precision level of astrometry, the dominant uncertainty is no longer measurement error, but systematic errors from the astrometric calibration. In particular, accurate knowledge of the focal plane distortion on the detector is required. The distortions of the PHARO 25 mas pixel ${ }^{-1}$ and NIRC2 20 mas pixel $^{-1}$ cameras was mapped to high precision in Metchev (2006). For NIRC2, this was done at the pre-ship testing stage in the laboratory. ${ }^{1} \mathrm{We}$ applied the respective distortion corrections to the measured pixel coordinates and calibrated the absolute pixel scales and orientations of the two detectors at each epoch from observations of WDS $18055+0230$ - a binary star with a well-known astrometric orbit from the Sixth Catalog of Orbits of Visual Binary Stars. ${ }^{2}$

The four epochs of astrometry spanning nearly 3 years confirmed HD 203030B as a common proper motion companion of

\footnotetext{
${ }^{1}$ See http://www2.keck.hawaii.edu/inst/nirc2/preship_testing.pdf.

2 See http://ad.usno.navy.mil/wds/orb6.html.
}

HD 203030A. At first epoch, HD 203030B was separated by $\rho=11^{\prime \prime} .923 \pm 0.021(487.1 \pm 1.8 \mathrm{AU})$ from the primary, at a position angle $\theta=108^{\circ} .76 \pm 0.12$. The changes in the position angle $(\theta)$ and separation $(\rho)$ between the pair at subsequent epochs are shown with the data points in Figure 2. The astrometric errors of the first-epoch measurement (from 2002 August 28) are added in quadrature to the errors of the measurements from the subsequent epochs. The solid line in each of the panels in Figure 2 represents the "association" line, synonymous with common proper motion. The dashed lines in the panels trace the expected change in the relative position of HD 203030B with respect to HD 203030A had the companion been a background star with a negligible apparent proper motion. The dotted lines on either side of the dashed lines represent the $1 \sigma$ uncertainties in the expected motion based on the Hipparcos proper-motion errors of the primary. HD 203030B is inconsistent with being an unrelated "stationary" background star at the $13 \sigma$ level. Because HD 203030 is not a member of a known open cluster, which could create the possibility of HD 203030B being just another kinematic member of the same cluster seen in projection, we assume that HD 203030A and B form a physical pair.

\subsubsection{Photometry}

Near-IR $H$ and $K_{S}$ magnitudes of HD 203030B were obtained from the individual unocculted $1.8 \mathrm{~s}$ exposures taken at Palomar Observatory during the first epoch of observations (2002 August 28 ). As the companion was not visible in the short $J$-band exposure, the $J$-band magnitude of HD 202030B was obtained from a deeper (50 s) $J$-band coronagraphic image of HD 203030 taken with Keck AO on 2005 July 14, in which the companion was detected.

The primary was saturated in the short Palomar exposures and was occulted by the coronagraph in the Keck $J$-band exposures, thus preventing a direct measurement of the magnitude difference between HD 203030 A and B. However, the night of 2002 August 28 was photometric, and we were able to calibrate the 
TABLE 2

Near-IR Magnitudes of HD 203030B

\begin{tabular}{ccccc}
\hline \hline$J$ & $H$ & $K_{S}$ & $\Delta K_{S}$ & $M_{K_{S}}$ \\
\hline
\end{tabular}

$18.13 \pm 0.55 \quad 16.85 \pm 0.12 \quad 16.21 \pm 0.10 \quad 9.56 \pm 0.10 \quad 13.15 \pm 0.14^{\mathrm{a}}$

a Assuming the Hipparcos parallax of $24.48 \pm 1.05$ mas for HD 203030A (Perryman et al. 1997).

near-IR photometry with respect to another program star-HD 13531 - unocculted $J H K_{S}$ images of which were obtained through the ND $1 \%$ filter on the same night and at the same air mass. HD 13531 is not known to be variable (its Hipparcos photometry is constant to within $0.015 \mathrm{mag}$; Perryman et al. 1997) and is therefore an adequate photometric standard. The near-IR transmissivity of the ND 1\% filter was established in Metchev \& Hillenbrand (2004). The $H$ - and $K_{S}$-band fluxes of HD 203030B and HD 13531 were measured in 0 "'65 diameter apertures (encompassing two Airy rings at $\left.K_{S}\right)$. Such wide apertures $(\approx 8$ PALAO PSFs across) were chosen to mitigate as much as possible the anisoplanatic distortion (5\%-10\% in FWHM) of the PALAO PSF toward the edge of the $25.7 \times 25.7$ PHARO field, and resulted in a high degree of photometric self-consistency ( $\mathrm{rms} \leq 0.05 \mathrm{mag})$. Experiments with smaller apertures, $0.18-0.36$ in diameter, with sizes proportional to the (variable) FWHM of the PSF, reproduced our wide-aperture photometry to within $0.10 \mathrm{mag}$ (although with a larger intrinsic scatter) without showing any systematic trends with aperture size. Estimates of the local background were obtained from the mean of the pixel counts in 0.25 wide annuli with inner radii between 0.50 and 1 ."25 (chosen to be at least 0.25 larger than the radius at which the stellar radial profile blended into the background). We used the 2MASS $H$ and $K_{S}$ magnitudes of HD 13531 to calibrate the transformation from instrumental $H$ and $K_{S}$ magnitudes to apparent magnitudes.

The $J$-band magnitude of HD 203030B was bootstrapped from the deeper NIRC2 images with respect to that of object 1 (Fig. 1). We used the same $00^{\prime \prime} 65$ diameter apertures, as for the $H$ and $K_{S}$ bands above. At $J$ band the poorer AO performance (Strehl ratio $\lesssim 10 \%$ ) and the much more severe PSF elongation due to anisoplanetism limited the accuracy of our measurements. All near-IR photometry of HD 203030B is listed in Table 2.

Finally, we note that HD 13531 itself was found to have a faint ( $\Delta J=4.84$ mag, $\Delta K_{S}=4.20$ mag) stellar proper-motion companion at an angular separation of 0 "'72 (Metchev 2006). The presence of the secondary (HD 13531B) affects the 2MASS magnitudes of the primary by $0.01 \mathrm{mag}$ at $J$ and by $0.02 \mathrm{mag}$ at $H$ and $K_{S}$, which we take into account. At its relatively large angular separation from HD 13531A (8-9 times the FWHM of the PALAO PSF), HD 13531B has a negligible effect on our photometric measurements.

\subsection{Spectroscopic Follow-up}

Spectroscopic observations of HD 203030B were obtained on 2005 July 14 with Keck AO after the association of the companion with the primary had been established. With NIRC2 we used the 20 mas pixel ${ }^{-1}$ camera in conjunction with the 80 mas wide slit and the low-resolution (LOWRES) grism for $R \sim 2000 K$-band spectroscopy. We obtained three 10 minute $K$-band spectra at an air mass of 1.03-1.06 with dithers along the slit between exposures. Conditions were photometric and the FWHM of the PSF as measured from the acquisition images was 50 mas. Telluric and instrumental features in the spectra were identified and corrected from observations of a nearby B9.5 V star (Grenier et al.
1999), HD 201859, obtained immediately afterward. Images of internal flat field and arc lamps were taken at the beginning of the night to calibrate the detector response and the wavelength dispersion of the system.

The individual frames were first flat-fielded and then pair-wise subtracted. The spectra of HD 203030B were then extracted using the IRAF task apall. We adopted an aperture width of 14 pixels $(0.28)$ centered on the spectrum of HD 203030B, which was traced by a high-order (6-10) polynomial. We estimated the background in 40 pixel $(0 " 80)$ wide bands centered $50-100$ pixels $\left(1{ }^{\prime \prime} 0-2\right.$ ". 0$)$ away from the object. The large widths of the slit and of the extraction aperture were chosen to minimize chromatic effects arising from the dependence of the FWHM of the AO PSF on wavelength (e.g., Goto et al. 2003). The success of this extraction approach was judged by the fact that three individual companion spectra extracted from wide apertures were consistent among one another, while variations in the AO PSF on timescales of minutes are expected to induce chromatic differences among spectra extracted from narrower apertures. Because HD 203030B is separated by almost $12^{\prime \prime}$ from HD 203030A, stray light from the primary was not an issue in the extraction of the companion spectrum. The spectra of the telluric standard were extracted in a similar fashion as those of HD 203030B, using the same aperture width, although with the background bands farther from the star to avoid contamination from the brighter PSF halo.

We calibrated the wavelength dispersion of the extracted spectra with the arc lamp spectra, and after interpolating over the intrinsic $\mathrm{Br} \gamma$ absorption in the telluric standard, we divided the extracted spectra of HD 203030B by those of the standard. We then median-combined the resulting three companion spectra and multiplied them by a 10,500 K blackbody. The final HD 203030B $K$-band spectrum was smoothed to match the instrumental resolution ( $R \approx 1300$, as measured from the FWHM of the arc lines) and is shown compared to spectra of known L6-T0 dwarfs in Figure 3.

\subsubsection{Spectral Type of HD $203030 B$}

The $K$-band spectrum of HD 203030B (Fig. 3) exhibits molecular absorption features due to $\mathrm{H}_{2} \mathrm{O}$ in the blue end and $\mathrm{CO}$ in the red end, as is typical of late-M to T0 dwarfs. No other molecular or atomic features are seen. In particular, the spectrum does not show either $\mathrm{Na}$ I doublet absorption at $2.21 \mu \mathrm{m}$, which is seen in the spectra of $\leq \mathrm{L} 2$ dwarfs at similar $(R \sim 2000)$ resolution, or $\mathrm{CH}_{4}$ absorption at $2.20 \mu \mathrm{m}$, characteristic of $\geq \mathrm{T} 0$ dwarfs (McLean et al. 2003). We thus infer that the spectral type of HD 203030B is intermediate between L2 and T0. For a more precise spectroscopic classification of HD 203030B we rely on $K$-band spectroscopic indices from the NIRSPEC Brown Dwarf Spectroscopic Survey (BDSS; McLean et al. 2003), designed to follow the L dwarf optical classification scheme of Kirkpatrick et al. (1999).

Following previous work on IR spectroscopic classification, McLean et al. (2003 and references therein) define three molecular indices in the $K$ band, measuring the onset of $\mathrm{H}_{2} \mathrm{O}, \mathrm{CH}_{4}$, and $\mathrm{CO}$ absorptions at $2.05,2.20$, and $2.30 \mu \mathrm{m}$, respectively. The indices are defined as the ratios of the median flux values in $40 \AA$ wide regions on either side of the molecular band head. They are therefore applicable to data with somewhat lower than the original $R \approx 2000$ resolution of the BDSS data and are well suited for our $R \approx 1300$ NIRC 2 spectrum. We consider only the $K$-band $\mathrm{CH}_{4}$ and $\mathrm{CO}$ indices of McLean et al., for which we measure values of $0.90 \pm 0.04$ and $0.85 \pm 0.03$, respectively, where the index means and their errors are obtained from measurements on the 


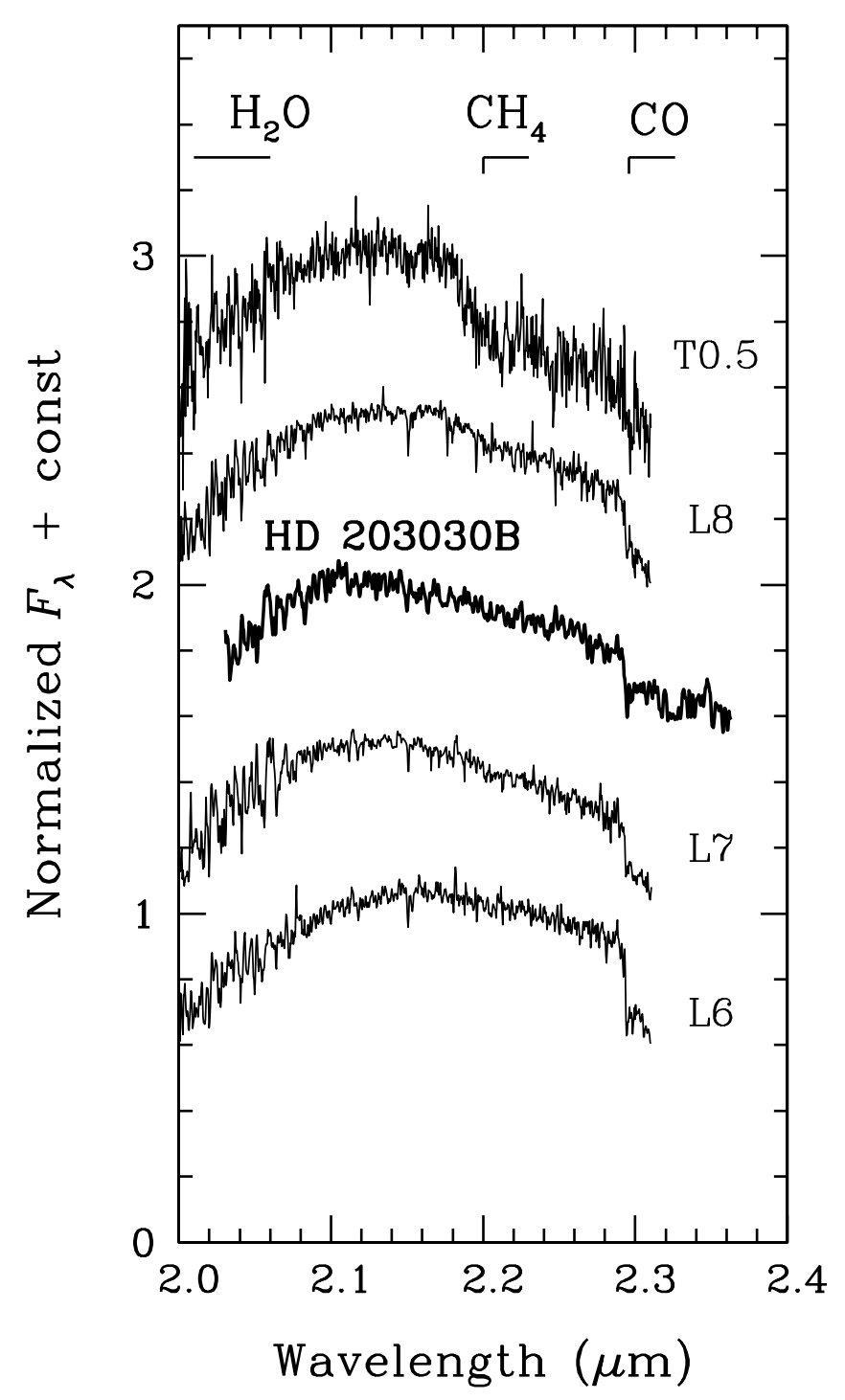

FIG. 3. $K$-band $R \approx 1300$ spectrum of HD 203030B obtained with Keck AO+NIRC2, compared to $R \approx 2000$ spectra of L6-T0 dwarfs from McLean et al. (2003). The spectra have been normalized to unity at $2.1 \mu \mathrm{m}$ and have been offset by a constant ( 0.5 in normalized flux) from each other for clarity. HD 203030B shows $\mathrm{H}_{2} \mathrm{O}$ and $\mathrm{CO}$ absorption, similar to other late $\mathrm{L}$ dwarfs, although it does not show the characteristic $\mathrm{CH}_{4}$ absorption of $\mathrm{T}$ dwarfs. The comparison dwarfs from McLean et al. (2003) are 2MASS 01033203+1935361 (L6), DENIS-P 020529.0$115925 \mathrm{AB}$ (L7), GL 584C (L8), and SDSS J083717.22-000018.3 (T0.5). [See the electronic edition of the Journal for a color version of this figure.]

three independent HD 203030B spectra. We do not use their $K$-band $\mathrm{H}_{2} \mathrm{O}$ index because it requires measurements blueward of $2.0 \mu \mathrm{m}$, where our spectrum of HD 203030B is cut off by the transmissivity of the NIRC2 LOWRES grism.

Comparing to data from McLean et al. (2003) and Cushing et al. (2005), we find that the $1 \sigma$ spectral-type ranges based on the $\mathrm{CH}_{4} \mathrm{~B}$ and $\mathrm{CO}$ indices are $\mathrm{L} 7-\mathrm{T} 0$ and $\mathrm{M} 7-\mathrm{L} 8$, respectively. As already noted in McLean et al. and Cushing et al., the $\mathrm{CO}$ index is of little use for spectral typing of ultracool dwarfs because it displays very little sensitivity to their effective temperatures. Hence, we use only the result from the $\mathrm{CH}_{4} \mathrm{~B}$ index. Having excluded the possibility that HD 203030B is a T dwarf, based on the lack of methane absorption at $2.20 \mu \mathrm{m}$, we adopt a final spectral type of L $7.5 \pm 0.5$ for it on the L dwarf classification scheme of Kirkpatrick et al. (1999) extending to L8 in the L dwarfs. If instead we adopt the L dwarf spectroscopic classification system of Geballe et al. (2002), based on lower resolution spectra but extending to $\mathrm{L} 9$ with the appropriately redefined $2.20 \mu \mathrm{m}$ methane index, the spectral type of HD 203030B is L7.5 \pm 1.5 (methane index value of $1.13 \pm 0.07$ ). Both classifications are consistent with (1) a visual comparison of the $K$-band spectrum of HD 203030B to the spectra of L6-T0 dwarfs (Fig. 3), (2) the near-IR colors of HD 203030B $(J-H=1.28 \pm 0.56 ; H-$ $K_{S}=0.64 \pm 0.16$; see Table 2), and (3) the empirical near-IR absolute magnitude versus spectral-type relations for $\mathrm{L}$ and $\mathrm{T}$ dwarfs from Kirkpatrick et al. (2000) and Vrba et al. (2004). Hence, the spectral type of HD 203030B is later than those of the coolest hydrogen-burning stars $(\sim \mathrm{L} 4)$, and it is a brown dwarf.

As we show in $\S 3.1$, HD 203030B is younger than typical field brown dwarfs. Hence, a lower than dwarf surface gravity may make its spectral classification with respect to field dwarfs inadequate. Our spectrum of HD 203030B does not allow us to assess its surface gravity empirically because it lacks features with well-expressed gravity sensitivity, the only one at $K$ band being the Na I $2.21 \mu \mathrm{m}$ doublet, which disappears after L2 in the L dwarfs (McLean et al. 2003). Models predict that at the age of the primary (130-400 Myr; see $\S 3.1)$ the surface gravity of HD $203030 \mathrm{~B}$ is $\approx 0.5$ dex lower than the gravities of $\sim 3$ Gyr aged field $\mathrm{L}$ dwarfs. This may affect the strength of the observed $\mathrm{CO}$ and $\mathrm{H}_{2} \mathrm{O}$ absorption, which are weakly sensitive to gravity. Enhanced absorption by water vapor that produces peaked $H$-band continua is indeed observed in younger, $<10-50 \mathrm{Myr}$ old, L dwarfs with low surface gravities (Lucas et al. 2001; Kirkpatrick et al. 2006). Our spectrum of HD 203030B does indeed exhibit a slightly peaked continuum shape near $2.1 \mu \mathrm{m}$-an effect not previously observed in L dwarfs. However, the effect of low surface gravity on $\mathrm{CO}$ and $\mathrm{H}_{2} \mathrm{O}$ absorption at $K$ band is likely too weak by the age of HD 203030B, as it is not distinctive even in the younger (1-50 Myr) early L dwarf 2MASS J014158234633574 (Kirkpatrick et al. 2006). Moreover, our estimate of the spectral type of HD 203030B does not depend on $\mathrm{CO}$ and $\mathrm{H}_{2} \mathrm{O}$ absorption indices, but on the $\mathrm{CH}_{4}$ index, which, in the lack of methane absorption, measures the slope of the continuum at $2.2 \mu \mathrm{m}$ - far from any $\mathrm{CO}$ and $\mathrm{H}_{2} \mathrm{O}$ band heads. The peaked appearance of our $K$-band spectrum near $2.1 \mu \mathrm{m}$ can, on the other hand, be traced to a possible chromatic effect in one of the telluric spectra at wavelengths $<2.1 \mu \mathrm{m}$. Therefore, we believe that our estimate of the spectral type of HD 203030B is not affected by its potentially lower surface gravity.

\section{PROPERTIES OF HD 203030B: AGE, LUMINOSITY, MASS, AND EFFECTIVE TEMPERATURE}

Fundamental substellar parameters are degenerate with age. Ages, masses, effective temperatures, and radii are empirically unconstrained in isolated brown dwarfs. Only substellar bolometric luminosities can be estimated from measurements of trigonometric parallaxes. The brown dwarf HD 203030B offers an opportunity to further resolve some of these degeneracies by the additional constraint on its age because of its association with HD 203030. Below we first discuss the factors that determine the age of the primary, and hence of the secondary, by assumption of coevality. We then use our knowledge of the age and the heliocentric distance of HD 203030 to obtain model-dependent estimates of the mass and effective temperature of its substellar companion.

\subsection{Age of the Primary}

HD 203030 is a G8 V star (Jaschek 1978), and its age can be constrained from a number of photometric and spectroscopic indicators established from $\mathrm{G}$ stars in well-studied open clusters 
of known ages. A probabilistic estimate of the age of HD 203030 can also be made based on comparison of its Galactic space motion to the space motions of known stellar populations and moving groups. The relevant stellar age indicators and the inferred ages are discussed below.

Position on the color-magnitude diagram.-HD 203030 has an apparent $V$-band magnitude of 8.48 mag (Eggen 1964) and a Hipparcos parallax of $24.48 \pm 1.05$ mas (Perryman et al. 1997). Thus, given an absolute $V$-band magnitude of 5.42 mag and a $B-V$ color of $0.75 \mathrm{mag}$ (Eggen 1964), HD 203030 sits on the main sequence and is between 0.1 and 10 Gyr old.

Stellar rotation.- Stellar rotation periods increase with age as a result of angular momentum transfer outward from the core to the envelope, and then via loss to stellar winds or to a circumstellar disk (e.g., Kraft 1970; Kawaler 1988; Pinsonneault et al. 1989). Koen \& Eyer (2002) find a photometric period of 4.1 days for HD 203030 in the Hipparcos data. This period lies in between the rotation periods of late $\mathrm{G}$ stars in the $\alpha$ Perseus Cluster (0.2-2.4 days; Prosser 1992) and in the Coma cluster and the Hyades (6-12 days; Radick et al. 1990), and is within the range of G-star rotation periods observed in the Pleiades (0.4-6 days; van Leeuwen et al. 1987; Krishnamurthi et al. 1998). Assuming that the photometric periodicity of HD 203030 is due to star spots and stellar rotation, the age of HD 203030 is between that of $\alpha$ Per (90 Myr; Stauffer et al. 1999) and that of the Hyades (625 Myr; Perryman et al. 1998), and possibly comparable to the age of the Pleiades (125 Myr; Stauffer et al. 1998).

Chromospheric $\mathrm{Ca}$ II $\mathrm{H}$ and $\mathrm{K}$ emission.-The strength of $\mathrm{Ca}$ II emission at 3968 and $3933 \AA$ in the cores of the $\mathrm{H}$ and $\mathrm{K}$ chromospheric absorption bands is a well-known measure of chromospheric activity and a proxy for the evolution of the stellar dynamo. A widely adopted indicator is the $R_{\mathrm{HK}}^{\prime}$ index, which measures the relative strength of $\mathrm{Ca}$ II emission with respect to the stellar continuum (Noyes et al. 1984). The value of the $R_{\mathrm{HK}}^{\prime}$ index for HD 203030 has been obtained from high-resolution optical spectra of the star by four separate groups, the individual measurements being: -4.20 (Strassmeier et al. 2000) ${ }^{3},-4.36$ (D. Soderblom 2000, private communication), -4.37 (Wright et al. 2004), and -4.47 (White et al. 2006). The mean value of the index from the four sources is $\log R_{\mathrm{HK}}^{\prime}=-4.35 \pm 0.06$ (standard error). Using an empirical age- $R_{\mathrm{HK}}^{\prime}$ relation from Donahue (1993):

$$
\log (t)=10.725-1.334 R_{5}+0.4085 R_{5}^{2}-0.0522 R_{5}^{3},
$$

where $t$ is the stellar age in years and $R_{5}=10^{5} R_{\mathrm{HK}}^{\prime}$, we calculate that the age of HD 203030 is $t=180_{-140}^{+260} \mathrm{Myr}$.

Coronal X-ray emission.-Similarly to chromospheric activity, coronal X-ray luminosity is another proxy for the strength of the stellar dynamo that can be applied to determine stellar ages (Vilhu 1984; Randich 2000). In a study of age diagnostics for young Sun-like (G and early K) stars, L. A. Hillenbrand et al. (2006, in preparation) find, based on an analysis of open cluster data, that the age of Sun-like stars is related to their X-ray luminosity following

$$
\log \left(t_{\mathrm{Myr}}\right)=-0.88 \log L_{\mathrm{X}}+33.88
$$

where $t_{\mathrm{Myr}}$ is the stellar age in megayears and $L_{\mathrm{X}}$ is the X-ray luminosity in ergs per second. The X-ray luminosity of HD 203030 from the ROSATAll-Sky Survey Faint Source Catalog (Voges et al.

\footnotetext{
3 Strassmeier et al. (2000) list $R_{\mathrm{HK}}$, which we convert to $R_{\mathrm{HK}}^{\prime}$ using the $B-V$ color of HD 203030 and an expression from Hartmann et al. (1984).
}

$2000)$ is $\log \left(L_{\mathrm{X}}\right)=29.01 \pm 0.16$, where we have converted count rates to flux using the conversion factor in Fleming et al. (1995). From this, we find that the age of HD 203030 is $220_{-90}^{+140} \mathrm{Myr}$, where the intrinsic scatter of relation (2), 0.16 dex (L. A. Hillenbrand et al. 2006, in preparation), has been added in quadrature to the error in $L_{\mathrm{X}}$.

Li I absorption.- - Lithium is destroyed in the convective envelopes of late-type stars, and so a high photospheric lithium abundance is a signature of stellar youth (Herbig 1965; Bodenheimer 1965). Strassmeier et al. (2000) measure a $\mathrm{Li}$ I 26708 equivalent width of $56 \mathrm{~m} \AA$ for HD 203030 in $R=25,000$ spectra. This is a factor of $\sim 2$ larger than the measured Li I equivalent widths in $R \sim 34,000$ spectra of stars of similar colors in the $625 \mathrm{Myr}$ old Hyades (Soderblom et al. 1990), a factor of $\sim 3$ smaller than the $\mathrm{Li}$ I equivalent widths in $R \sim 20,000$ spectra of late $\mathrm{G}$ stars in the 125 Myr old Pleiades (Duncan \& Jones 1983), and is within the range of $\mathrm{Li}$ I equivalent widths in $R \sim 48,000$ spectra of analogous stars in the M 34 (NGC 1039) open cluster (250 Myr; Jones et al. 1997). HD 203030 is therefore younger than $625 \mathrm{Myr}$, probably older than $125 \mathrm{Myr}$, and likely of order $250 \mathrm{Myr}$ old.

UVW Galactic space motion.-Young stars inherit the kinematics of their birth environment for tens to hundreds of megayears before their space motion is disrupted by dynamical interactions with the Galactic environment. The Galactic velocity vector of a star can therefore be used to obtain a probabilistic estimate of the star's age, when compared to the space motions of comoving groups of stars with known ages. Based on its Galactic space motion $\left(U=-23.4 \mathrm{~km} \mathrm{~s}^{-1}, V=-15.9 \mathrm{~km} \mathrm{~s}^{-1}, W=-12.2 \mathrm{~km} \mathrm{~s}^{-1}\right)$, Montes et al. (2001) classify HD 203030 as a probable kinematic member of the IC 2391 supercluster, with an estimated age of 35-50 Myr (Mermilliod 1981; Barrado y Navascués et al. 2004).

To summarize, based on its chromospheric and coronal activity, HD 203030 is $\sim 250$ Myr old, with a possible age range of 130-400 Myr. This age is fully consistent with its stellar rotation rate and photospheric lithium absorption and is also supported by its main-sequence position in a color-magnitude diagram. However, this age does not agree with the young kinematic age of HD 203030 quoted in Montes et al. (2001). Nevertheless, such discrepancies are not unusual in kinematically selected samples of young stars. Indeed, we note that the $U V W$ velocity vector of HD 203030 inhabits an intermediate position between the loci of the 20-150 Myr Local Association, the 100-300 Myr Castor moving group, and the 600 Myr Hyades supercluster (Fig. 1 in Montes et al. 2001, and references therein). If HD 203030 were a member of the Local Association or the Castor moving group, instead of the IC 2391 moving group, its kinematic age would be consistent with the ages derived from the other criteria.

\subsection{Luminosity}

We determine the absolute $K_{S}$-band magnitude of HD 203030B (Table 2) by assuming that it is at the same heliocentric distance as the primary (Hipparcos parallax $24.48 \pm 1.05$ mas; Perryman et al. 1997). To convert the absolute magnitude to bolometric luminosity, we use a calibration of the $K$-band bolometric correction $\mathrm{BC}_{K}$ versus $\mathrm{L}-\mathrm{T}$ spectral type from Golimowski et al. (2004). We take into account that the calibration of Golimowski et al. (2004) is for the spectral classification system of Geballe et al. (2002) on which the spectral type of HD 203030B is $\mathrm{L} 7.5 \pm 1.5$ (§ 2.2.1). We also note that the $\mathrm{BC}_{K}$ versus spectraltype relation from Golimowski et al. is for the MKO near-IR filter set, while our data are on the CIT filter set. However, the synthetic transformations of Stephens \& Leggett (2004) show that the MKO $K$ and CIT $K_{S}$ filters produce nearly identical results 
with an offset of 0.00-0.02 mag for L6-L9 dwarfs. This small offset has been added to our error budget. Finally, we note that the bolometric corrections in Golimowski et al. are compiled from data for $>1$ Gyr old, high-surface gravity field dwarfs and may need to be corrected for the expected $\approx 0.5$ dex lower surface gravity of the $\sim 250 \mathrm{Myr}$ old HD $203030 \mathrm{~B}$. The sense of this correction is unknown empirically, as the body of data on young $\mathrm{L}$ dwarfs is extremely limited. From the models of Burrows et al. (1997, 2001) and Chabrier et al. (2000) we infer that the magnitude of the correction over a 0.5 dex change in surface gravity is $\leq 0.05$ mag and that it may be either positive or negative. Therefore, we treat it as an added error term.

Averaging the bolometric corrections for the six L6-L9 dwarfs in Table 6 of Golimowski et al. (2004), we find $\mathrm{BC}_{K}=3.19 \pm$ $0.11 \mathrm{mag}$ for HD 203030B. The bolometric magnitude of HD $203030 \mathrm{~B}$ is then $M_{\mathrm{bol}}=M_{K_{S}}+\mathrm{BC}_{K}=16.34 \pm 0.18 \mathrm{mag}$ and, assuming $M_{\mathrm{bol}, \odot}=4.74 \mathrm{mag}$ for the Sun (Drilling \& Landolt 2000), its luminosity is $\log \left(L / L_{\odot}\right)=-4.64 \pm 0.07$. The bolometric luminosity of HD 203030B is thus comparable to those of single-field L6-T5 dwarfs [mean $\log \left(L / L_{\odot}\right)=-4.58$ with a standard deviation of 0.15 dex; see Table 6 in Golimowski et al.]. That is, at the 130-400 Myr age of HD 203030B, transition L/T dwarfs are not more luminous than their $>1$ Gyr aged field counterparts. This is an unusual but significant result, given that at its $\approx 0.5$ dex lower anticipated surface gravity the luminosity of HD 203030B is expected to be $\approx 0.5$ dex higher than that of older $\mathrm{L} / \mathrm{T}$ dwarfs in the field (provided that the effective temperature at the $\mathrm{L} / \mathrm{T}$ transition is independent of age). As we discuss in the following sections, this translates in an unusually low effective temperature for HD 203030B ( $\S 3.3$ ), and indicates either a necessity to reevaluate the effective temperatures and ages of $\mathrm{L} / \mathrm{T}$ dwarfs in the field or a gravity dependence in the spectral type-effective temperature relation near the $\mathrm{L} / \mathrm{T}$ transition $(\S 4.2)$.

\subsection{Effective Temperature}

The effective temperature of HD 203030B can be inferred from its age and bolometric luminosity by adopting an agedependent theoretical estimate of its radius. Using the age and luminosity values obtained above, the models of Burrows et al. (1997) predict a mean radius of $1.06 R_{\mathrm{J}}$ (at the mean age and luminosity of HD $203030 \mathrm{~B}$ ) with a range of $1.00-1.22 R_{\mathrm{J}}$ (corresponding to the full age range and $1 \sigma$ luminosity range of $\mathrm{HD}$ 20303B), and a mean effective temperature of $1206 \mathrm{~K}$ with a range of 1090-1280 K. These values and their ranges are also consistent with those inferred from the models of Chabrier et al. (2000; DUSTY) and Baraffe et al. (2003; COND).

Alternatively, the effective temperature of HD 203030B can be inferred from its spectral type, by comparison to the compilation of spectral type and effective temperature data for brown dwarfs with known parallaxes in Vrba et al. (2004) and Golimowski et al. (2004). This seemingly empirical approach is also model dependent because, despite the use of measured bolometric luminosities, Vrba et al. (2004) and Golimowski et al. (2004) also adopt brown dwarf radii based on theoretical evolutionary models (from Burrows et al.). Nevertheless, because these authors use only ensemble mean values for the radii $\left(0.90 \pm 0.15 R_{\mathrm{J}}\right.$; Vrba et al. 2004) or the ages (3 Gyr; Golimowski et al. 2004) of field brown dwarfs, their results do not hinge on the details of the theoretical models. From 12 distinct L dwarfs listed in Table 7 of Vrba et al. (2004) and Table 6 of Golimowski et al. (2004) we find that the effective temperatures of $\mathrm{L}$ dwarfs of spectral types L7 or later (i.e., consistent with that of HD 203030B) fall in the range $1330-1650 \mathrm{~K}$, with a mean of $1442 \pm 103 \mathrm{~K}$ (where we have excluded the temperature estimate for SDSS J042348.57-

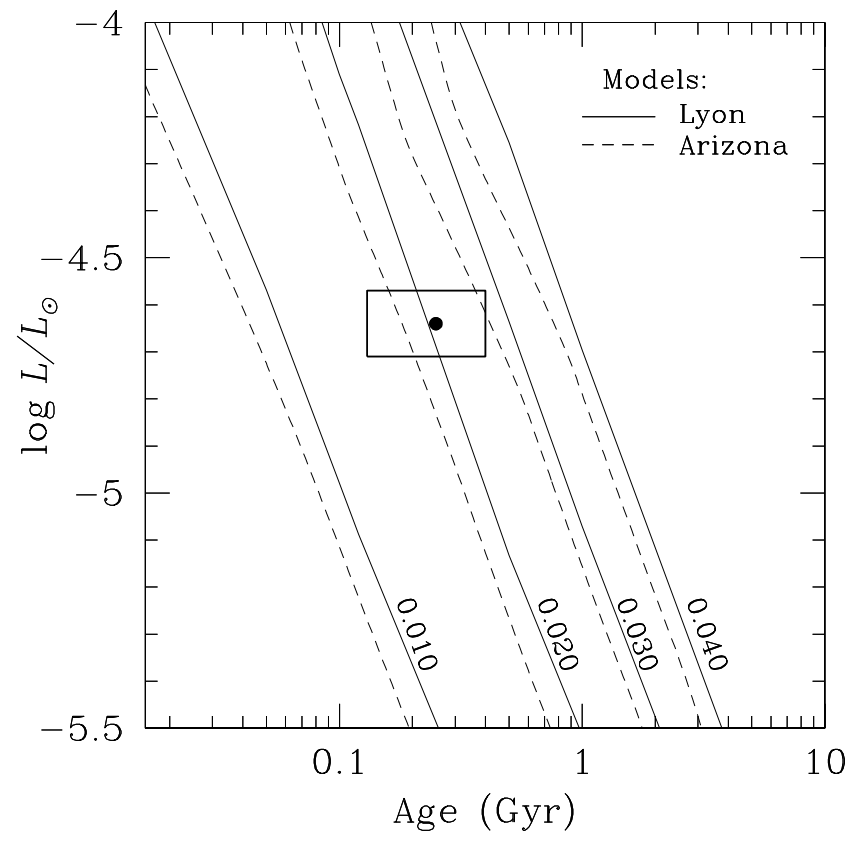

FIG. 4.-Comparison of the luminosity and age of HD 203030B to predictions for $0.010-0.040 M_{\odot}$ brown dwarfs from the evolutionary models of Baraffe et al. (2003; solid lines) and Burrows et al. (1997; dashed lines). The estimated mass of the substellar companion is between 0.012 and $0.031 M_{\odot}$.

041403.5, now resolved into a L6/T2 binary; Burgasser et al. $2005 \mathrm{~b}$ ). The individual estimates have errors of approximately $100-200 \mathrm{~K}$, arising from a combination of uncertainties in the photometry, parallaxes, and adopted radii or ages of the brown dwarfs.

We note that the two ranges obtained for the effective temperature of HD 203030B, 1090-1280 K, based on its luminosity and the assumption of a model-dependent radius, and 1330-1650 K, from its spectral type and a comparison with the semi-empirical calibrations of Vrba et al. (2004) and Golimowski et al. (2004), are only marginally consistent with each other, with HD 203030B appearing on average $240 \mathrm{~K}$ cooler. The lower inferred effective temperature of HD 203030B is a direct consequence of the assumption of a $\approx 15 \%$ larger radius (because of its young age) and similar bolometric luminosity ( $\$ 3.2$ ) compared to late L dwarfs in the field.

\subsection{Mass}

We estimate the mass of HD 203030B using the substellar evolution models from the Lyon (Chabrier et al. 2000; Baraffe et al. 2003) and Arizona (Burrows et al. 1997, 2001) groups. Figure 4 shows the allowed locus of values (thick rectangle) around the adopted age and luminosity ( filled dot) of HD 203030B overlaid on the evolutionary tracks. We have only plotted the COND suite of models (Baraffe et al. 2003) from the Lyon group, as the evolution of substellar luminosity predicted by the DUSTY (Chabrier et al. 2000) and COND models is nearly identical (Baraffe et al. 2003). Based on these predictions, the mass of HD 203030B is between 0.012 and $0.031 M_{\odot}$, with the adopted mean luminosity and age values corresponding to $0.023 M_{\odot}$. If, instead, we estimate the mass of HD 203030B from the effective temperature $(1442 \pm 103 \mathrm{~K})$ corresponding to its spectral type from the calibrations of Golimowski et al. (2004) and Vrba et al. (2004), then its mass would be $0.032_{-0.012}^{+0.009} M_{\odot}$. However, the predicted bolometric luminosity of HD $203030 \mathrm{~B}$ in this case would be a factor of 1.3-2.3 greater than the inferred one.

At a mass ratio of only $q=M_{2} / M_{1} \approx 0.02$, the HD 203030 $\mathrm{A} / \mathrm{B}$ system is among the lowest mass ratio binaries discovered 
through direct imaging to date, along with HR $7329 \mathrm{~A} / \mathrm{B}$ (Lowrance et al. 2000), AB Pic A/B (Chauvin et al. 2005b), and GQ Lup A/B (Neuhäuser et al. 2005). All of these very low mass ratio $(q \leq 0.03)$ binaries are young $(\leq 400 \mathrm{Myr})$ and have projected separations $>100 \mathrm{AU}$, with estimated orbital periods near the $\sim 10^{5}$ day peak of the G-star binary period distribution of Duquennoy \& Mayor (1991). Wide very low mass ratio systems may thus not be highly unusual at young ages and will be important for understanding the architecture of planetary systems in conjunction with their closer-in counterparts from radial velocity surveys.

\section{THE EFFECTIVE TEMPERATURE AT THE L/T TRANSITION}

The uncharacteristically low effective temperature of the young HD 203030B sets it apart from older L7-L8 field dwarfs with known parallaxes. Because the age of HD 203030B is known, unlike the ages of field brown dwarfs, this result has the potential of providing an important constraint for understanding the physics of the L/T transition. However, prior to delving into the implications, we critically consider all factors underlying our estimate of the effective temperature of HD 203030B.

\subsection{Reliability of the Effective Temperature Estimate}

Our estimate of the effective temperature of HD 203030B hinges on the following assumptions and factors:

1. physical association and coevality of HD 203030A and B;

2. accuracy of the photometry of HD 203030A, B, and of the reference HD 13531A, or variability in any of the above;

3. accuracy of the spectral classification;

4. age of HD 203030A;

5. heliocentric distance of HD 203030A;

6. unknown multiplicity of HD 203030B;

7. bolometric correction for HD 203030B;

8. accuracy of the theoretical evolutionary models.

We critically address each of these issues below:

1. If HD $203030 \mathrm{~B}$ were older than the primary, the theoretical estimate of its radius could be sufficiently small $\left(\lesssim 0.9 R_{\mathrm{J}}\right)$ to drive its mean effective temperature (corresponding to its mean age and luminosity; $\S \S 3.1,3.2$ ) above $1330 \mathrm{~K}$ and to make it consistent with the effective temperatures of L/T dwarfs in the field. However, the physical association of HD 203030B with its primary was already demonstrated beyond reasonable doubt in $\S 2.1$. While the possibility remains that HD 203030B is an old field brown dwarf captured by a younger HD 203030A, it is highly unlikely.

2. An overestimate of the apparent brightness of HD 203030B by $\geq 0.3$ mag would be sufficient to resolve the effective temperature discrepancy. This remains a possibility, especially given that precise ( $\leq 0.1 \mathrm{mag})$ AO photometry is notoriously difficult to obtain because of variations in the quality of the AO PSF on timescales of seconds. Our observations on 2002 August 28 benefitted from photometric conditions and relatively stable $\mathrm{AO}$ performance, with $K_{S}$-band Strehl ratios ranging between $40 \%$ and $60 \%$. Furthermore, our choice of wide $(\approx 8$ FWHM) photometric apertures alleviated the effect of variations in the total enclosed power that might be caused by changes in the AO correction. Both of these factors contributed to the high degree of self-consistency of our $K_{S}$-band AO photometry: $\leq 0.05$ mag among individual exposures $(\S 2.1 .2)$. We therefore believe that a systematic error in our photometry is not the culprit for the inferred discrepancy in the effective temperature.

Even though the photometry may be accurate, any of HD 203030A, HD 203030B, or HD 13531A (the photometric ref- erence) could be variable by $\geq 0.3 \mathrm{mag}$ at $K_{S}$ and thus possibly account for the fact that HD $203030 \mathrm{~B}$ appears cooler than expected (because of being underluminous). Indeed, HD 203030A and HD 13531A are both young $(\leq 0.5 \mathrm{Gyr})$ stars and therefore expected to be variable to some extent. Furthermore, variability amplitudes as high as 0.5 mag have been observed in L/T transition dwarfs on timescales of 0.3-3 hr (Enoch et al. 2003). However, as already pointed out ( $\S 2.1 .2$ ), the Hipparcos photometry of HD 13531 shows no variability; hence HD 13531 appears to be an adequate photometric standard. In addition, our differential photometry between HD 203030B and object 1 from the individual $60 \mathrm{~s} K_{S}$-band coronagraphic exposures spanning $1.3 \mathrm{hr}$ on 2002 August 28 does not exhibit deviations larger than $0.1 \mathrm{mag}$ from the mean (rms scatter $=0.06 \mathrm{mag}$ ). Barring the highly unlikely scenario in which HD 203030B and object 1 follow photometric variations of similar magnitudes, periods, and phases, we infer that HD 203030B is not strongly variable, and therefore its low effective temperature cannot be explained by a period of low intrinsic luminosity.

3. Spectral classification of L dwarfs in the near-IR is still not as well established as in the visible. Optically typed L dwarfs do not follow a unique and monotonic spectral sequence in the nearIR, indicating that the two wavelength regions probe different photospheric physics (Kirkpatrick 2005). It is therefore possible that the spectral type of HD 203030B inferred from its $K$-band spectrum (Fig. 3) does not correspond to the same spectral type in the visible. However, there is very little room for error in the present spectral classification. On one hand, the spectral type of HD 203030B is not later than L8 (on the L dwarf classification scheme of Kirkpatrick et al. 1999); i.e., it is not a T dwarf, because its $K$-band spectrum lacks methane absorption at $2.20 \mu \mathrm{m}(\S 2.2 .1)$. On the other hand, if the spectral type of HD 203030B were earlier than L7, its low effective temperature would be even more discrepant with those of similarly typed field L dwarfs. We are therefore confident that our $K$-band spectral classification of HD $203030 \mathrm{~B}$ is accurate to the claimed precision.

4. An older age for HD 203030 would imply a longer contraction time for the substellar companion and hence a smaller radius. Using the models of Burrows et al., we find that given the measured bolometric luminosity, an age $>1$ Gyr would be required at to make the brown dwarf radius sufficiently small $\left(\lesssim 0.9 R_{\mathrm{J}}\right)$ to raise its effective temperature above $1330 \mathrm{~K}$. However, as discussed in $\S 3.1$, all age-dating criteria indicate that HD 203030 is younger than the Hyades (625 Myr).

5. If HD 203030 were at a larger heliocentric distance, HD 203030B would be intrinsically more luminous and hence hotter. A $13 \%$ error in the Hipparcos parallax could account for the observed discrepancy in effective temperatures. We note that this would be of the same relative magnitude and sense as the error in the Hipparcos distance to the Pleiades (Pinsonneault et al. 1998). It is possible that such systematic zonal errors of similar magnitude exist also in other parts of the sky (e.g., the Coma cluster; Pinsonneault et al. 1998). However, the inferred Hipparcos systematic error leading to the Pleiades distance problem is of order 1 mas. This would result in only a $\approx 4 \%$ error in the parallax (24.48 mas) of HD 203030 - too small to explain the effective temperature discrepancy.

6. The existence of an unresolved binary companion to HD 203030B, a hypothetical HD 203030 "C" at $\lesssim 4$ AU from the secondary, would change the inferred bolometric luminosity of the secondary. Indeed, previously unknown binarity at the L/T transition has been recently invoked as a possibility in explaining the observed brightening in $J$ absolute magnitude from late $\mathrm{L}$ to early T spectral types (Burgasser et al. 2005b; Liu et al. 2005). 
However, a companion to HD 203030B would have the opposite effect: it would decrease the bolometric luminosities of the individual components (HD 203030B and "C"), without much affecting their spectral types, and would thus exacerbate the problem.

7. In estimating the absolute $K_{S}$-band magnitude of HD 203030B we used bolometric corrections from Golimowski et al. (2004). The accuracy of the bolometric corrections is limited by the presently small amount of data on $\mathrm{L}$ and $\mathrm{T}$ dwarfs and by the even smaller body of knowledge of the non-Raleigh-Jeans IR spectral energy distributions of brown dwarfs at $>3 \mu \mathrm{m}$. Only recently has Spitzer IRS spectroscopy over 5.5-38 $\mu \mathrm{m}$ confirmed that the bolometric corrections of Golimowski et al. are accurate to within $0.10 \mathrm{mag}$ (Cushing et al. 2006), albeit for a small number of $\mathrm{L}$ and $\mathrm{T}$ dwarfs. Regardless, systematic uncertainties in the $K$-band bolometric corrections of $\mathrm{L}$ and $\mathrm{T}$ dwarfs do not affect our comparison of effective temperatures in relative terms, because both studies that calibrate $\mathrm{L}$ and $\mathrm{T}$ spectral types versus effective temperature (Vrba et al. 2004; Golimowski et al. 2004) use the same scale of bolometric corrections as the one adopted by us.

8. Finally, our calculation of the effective temperature for HD 203030B hinges on the accuracy of the substellar evolutionary models of Burrows et al. (1997, 2001). Without further observational constraints (e.g., dynamical mass, surface gravity) it is not possible to empirically test the accuracy of these theoretical predictions. However, the radii of the brown dwarfs in the studies of Vrba et al. (2004) and Golimowski et al. (2004) are also estimated based on these models. Our approach in estimating the effective temperature of HD 203030B from its bolometric luminosity and age is therefore fully consistent with the methods employed in these two studies.

Having considered and rejected all possible factors that may have lead us to underestimate the effective temperature of HD 203030B $\left(1206_{-116}^{+74} \mathrm{~K}\right)$ in comparison to the temperature expected from its $\mathrm{L} 7.5 \pm 0.5$ spectral type $(1442 \pm 103 \mathrm{~K})$, we conclude that HD $203030 \mathrm{~B}$ is indeed cooler than late L dwarfs in the field.

\subsection{Origin of the Temperature Discrepancy}

We pointed out in $\S 4.1$ that the discrepantly low effective temperature of HD 203030B arises mostly from the larger substellar radius adopted for HD 203030B, because of its younger age but similar luminosity compared to older brown dwarfs of the same spectral type. Having established the robustness of our age and luminosity determinations of HD 203030B ( $\S 4.1$ ), we conclude that either (1) the effective temperatures of brown dwarfs across the L/T transition in Golimowski et al. (2004) and Vrba et al. (2004) have been overestimated on average by $240 \mathrm{~K}$ because of unresolved binarity, (2) the ages of L/T transition dwarfs in the field have been overestimated or, equivalently, their radii underestimated (and hence their effective temperatures overpredicted) by theoretical models, or (3) the range of effective temperatures over which the $\mathrm{L} / \mathrm{T}$ transition occurs extends to cooler temperatures than found in the empirical studies of Golimowski et al. (2004) and Vrba et al. (2004) due, e.g., to variations in surface gravity. We address each of these possibilities in turn below.

The effective temperatures of field brown dwarfs at the L/T transition may have been overestimated if the majority of these brown dwarfs are unresolved close binaries, i.e., if they appear overluminous. Indeed, Burgasser et al. (2005b) find that $~ 50 \%$ of late $\mathrm{L}$ to early $\mathrm{T}$ dwarfs turn out to be binaries when imaged at high angular resolution (e.g., with $\mathrm{AO}$, or with $H S T$ ), including one of the L/T brown dwarfs considered in the studies of Golimowski et al. (2004) and Vrba et al. (2004), SDSS J042348.57-041403.5. We test whether unresolved binarity is a factor by focusing only on these L/T transition dwarfs that have been imaged at high angular resolution. Seven of the L7-L8 dwarfs in Vrba et al. have a priori known multiplicity from HST WFPC2 (Bouy et al. 2003; Gizis et al. 2003), i.e., they have either been resolved into binaries, or have remained unresolved. The mean effective temperature of these is $1459 \pm 102 \mathrm{~K}$. It is similar to, if not somewhat higher than, the mean effective temperature, $1403 \pm 117 \mathrm{~K}$ (both sets of errors denote standard deviations), of the remaining four L7-L8 dwarfs that have not been observed at high angular resolution. Therefore, it is unlikely that unresolved binarity has lead to a significant overestimate of the effective temperatures across the L/T transition.

The second hypothesis (overestimated ages leading to underestimated radii) is not directly testable with the existing observational data, as the radii of $\mathrm{L}$ and $\mathrm{T}$ dwarfs have not been measured empirically. Nevertheless, following our discussion in $\S 3.3$, we construct an indirect test by comparing the effective temperatures of all known L7-L8 dwarf companions to stars (with known ages and distances) to the effective temperatures of field late $\mathrm{L}$ dwarfs with known trigonometric parallaxes from the studies of Golimowski et al. and Vrba et al. Given a self-consistent application of the theoretical models (Burrows et al. 1997, 2001), the two sets of late L dwarfs, companions versus free-floating, should have identical effective temperatures. The inferred model-dependent effective temperatures of all L7-L8 companions to stars (GJ 584C, Kirkpatrick et al. 2001; GJ 337C, Wilson et al. 2001; Burgasser et al. 2005a; HD 203030B, this paper) are all $\leq 1345 \mathrm{~K}$, which is $\geq 100 \mathrm{~K}$ below the mean for field L7-L8 dwarfs with known trigonometric parallaxes ( $\S 3.3$; Golimowski et al. 2004; Vrba et al. 2004). While the corresponding deviation in each case is only $1-2 \sigma$, the statistical significance of the overall effect is $>99.5 \%$ (i.e., $\geq 3 \sigma$ ). If the effect is real, it indicates that the predicted radii of field late L dwarfs are $10 \%-15 \%$ too small (making their effective temperatures too high) compared to those of the late L companions. Equivalently, this translates into a factor of a 1.5-2 overestimate of the adopted ages for late $\mathrm{L}$ dwarfs in the field, according to the models. Indeed, the mean ages of the three L7-L8 companions (ranging between 0.3 and 2.0 Gyr) are a factor of $\gtrsim 1.5$ smaller than the 2.9 Gyr mean statistical age of late L dwarfs in the field (Allen et al. 2005; also based on models from Burrows et al. 2001). While the youngest of the late L companions, HD 203030B, was the product of a targeted search for brown dwarfs around $\leq 0.5$ Gyr old stars (Metchev \& Hillenbrand 2004; Metchev 2006), and so should be excluded from this age comparison, GJ 584C and GJ 337C were not. Thus, barring a systematic underestimate of the mean ages of these two stellar primaries by a factor of $\sim 1.5$ (not at all unlikely, given that their maximum ages are 2.5 and $3.4 \mathrm{Gyr}$, respectively; Kirkpatrick et al. 2001; Wilson et al. 2001), it is possible that the ages of field late $\mathrm{L}$ dwarfs have been overestimated by a factor of $\sim 1.5$. A similar age overestimate is inferred when using the models of Chabrier et al. (2000) and Baraffe et al. (2003). That is, the result does not hinge on peculiarities in either set of models. Thus, substellar cooling rates may have been underpredicted, or effective temperatures in the initial conditions may have been overestimated, in both the Arizona and the Lyon models. However, given the strong reliance of this result on the ages of two isolated stars, which are not determined as accurately as the ages of stars in stellar associations, we cannot claim with certainty to have discovered any inaccuracy in the theoretical models. The hypothesis needs to be revisited once accurate bolometric luminosities are determined for a larger number of substellar objects with well-known ages, e.g., in open clusters.

The final hypothesis (that the L/T transition extends to cooler temperatures) is the default one, as it reconciles previous determinations 
of the effective temperature at the L/T transition with the present data, without seeking constraints on the underlying physics. A physical interpretation may nonetheless focus on the role of metallicity and surface gravity in the L/T transition (e.g., Burrows et al. 2006). The metallicity of HD 203030B may be assumed to be the same as that of the primary, for which Nordström et al. (2004) measure $[\mathrm{Fe} / \mathrm{H}]=-0.01$, i.e., solar, and likely not very different from that of most field L dwarfs. However, as already discussed ( $(2.2 .1)$, the gravity of the $\sim 250$ Myr old HD 203030B is expected to be $\approx 0.5$ dex lower than the gravities of $>1$ Gyr old field brown dwarfs, corresponding to its $10 \%-15 \%$ larger adopted radius. While we determined that gravity has not affected our estimates of the effective temperature and luminosity of HD 203030B in a systematic way, its effect on photospheric chemistry in brown dwarfs is unknown. We note that, among stars, late-type giants have 200-600 K lower effective temperatures than dwarfs of the same spectral type (see Gray 1992; Dyck et al. 1996). Hence, a similar trend among substellar objects is not at all unlikely. A dependence on surface gravity in the effective temperature at which the $\mathrm{L}$ dwarf sequence merges onto the $\mathrm{T}$ dwarf sequence is indeed predicted by the "unified cloudy model" of Tsuji \& Nakajima (2003). These authors propose such a dependence to explain the observed brightening of early $\mathrm{T}$ dwarfs at $J$ band (Dahn et al. 2002; Tinney et al. 2003; Vrba et al. 2004), arguing that the brighter early $\mathrm{T}$ dwarfs have lower surface gravities because they are younger. We note that Tsuji \& Nakajima's theory does not agree with the fact that the young HD 203030B is not brighter than its older late L counterparts in the field ( $\S 3.2)$. Furthermore, Liu et al. (2006) and Burgasser et al. (2006) have recently demonstrated that the $J$-band brightening across the L/T transition is not an age-spread effect, but a real feature of brown dwarf evolution, as might be explained by disruption of condensate clouds at a fixed effective temperature (Ackerman \& Marley 2001; Burgasser et al. 2002). Still, with HD 203030B we have now shown that the effective temperatures of different $\mathrm{L} / \mathrm{T}$ transition objects may not be identical. It therefore remains an open question whether surface gravity may have a role in setting the effective temperature at the L/T transition. Planned optical and $J$-band spectroscopy of HD 203030B, combined with theoretical modeling of gravitysensitive alkali, metal-hydride, and VO features will attempt to address this issue. Unfortunately, the magnitude of the effect that we will be trying to measure is of order of the accuracy with which modern models of substellar photospheres can match gravitysensitive features in brown dwarf spectra. The role of gravity on the effective temperatures of L dwarfs at a fixed spectral type may thus be better addressed with younger (albeit earlier type) objects, such as the recently discovered 2MASSW J1207334-393254B (Chauvin et al. 2005a) and 2MASS J01415823-4633574 (Kirkpatrick et al. 2006).

\section{CONCLUSION}

We have discovered a proper-motion substellar companion to the 130-400 Myr old Sun-like star HD 203030. The companion, HD 203030B, is 11".9 away from the primary, at a projected orbital separation of 487 AU. Assuming coevality with the primary star, substellar evolution models place the companion mass at $0.023_{-0.011}^{+0.008} M_{\odot}$. From $K$-band AO spectroscopy, we determine a spectral type of L $7.5 \pm 0.5$ for HD 203030B. The brown dwarf is thus near the spectroscopic L/T transition, characterized by diminishing amounts of dust in substellar photospheres. Because of its association with a main-sequence star of a known age and heliocentric distance, HD 203030B offers a rare chance to probe the $\mathrm{L} / \mathrm{T}$ transition in a setting in which most of the degeneracies characteristic of substellar models are resolved.

We find that the bolometric luminosity of HD 203030B is comparable to that of $>1$ Gyr old field dwarfs of similar spectral types, despite the $\approx 0.5$ dex lower gravity anticipated at the young age of HD 203030B. As a result, theoretical models of substellar evolution predict $\mathrm{a} \approx 240 \mathrm{~K}$ cooler effective temperature for HD 203030B compared to the effective temperatures of its older counterparts in the field. We consider three hypotheses for the discrepancy: (1) that the bolometric luminosities and effective temperatures of late $\mathrm{L}$ dwarfs in the field have been overestimated because of unresolved binarity; (2) that the effective temperatures of late L dwarfs in the field may have been overestimated (equivalently, their radii underestimated) because theoretical models may have overpredicted their ages; and (3) that the spectral typeeffective temperature for late $\mathrm{L}$ dwarfs is not age and gravity independent. Based on multiplicity studies of field brown dwarfs from high-angular resolution observations with HST and AO, we do not find a significant discrepancy between the effective temperatures (or luminosities) of brown dwarfs with known and unknown multiplicities, and we therefore rule out the first possibility. We test the second hypothesis by comparing the effective temperatures of late $\mathrm{L}$ secondaries of main-sequence stars (with known ages and distances) to late $\mathrm{L}$ dwarfs in the field. We find evidence that the late L companions are $\geq 100 \mathrm{~K}$ cooler and $\sim 1.5$ times younger than their isolated counterparts. However, because ages of individual stars cannot be claimed with such accuracy, the significance of this result is only marginal. The final hypothesis is a compromise between the discrepant effective temperatures of HD 203030B and late L dwarfs in the field and draws a parallel with the dependence of spectral type and effective temperature on surface gravity in stars (Gray 1992). Both remaining hypotheses await testing from larger samples of brown dwarfs with known distances and ages, either as members of known stellar associations or as companions to stars.

The authors acknowledge many insightful discussions with Travis Barman, J. Davy Kirkpatrick, and Ian McLean during the preparation of the manuscript. We are grateful to our support astronomers and engineers Randy Campbell, Rick Burress, and Jeff Hickey for their guidance in using Keck and Palomar AO, to Keith Matthews and Dave Thompson for advice with NIRC2 spectroscopy, and to our telescope operators at the Palomar and Keck II telescopes, Jean Mueller, Karl Dunscombe, and Cynthia Wilburn. We also wish to extend special thanks to those of Hawaiian ancestry, on whose sacred mountain of Mauna Kea we are privileged to be guests. Without their generous hospitality, the spectroscopic observations presented herein would not have been possible. Support for S. A. M. was provided by NASA through the Spitzer Fellowship Program, under award 1273192. This work was supported by NASA grant NNG05GJ37G and NASA/JPL contract 1224566 .

\section{REFERENCES}

Ackerman, A. S., \& Marley, M. S. 2001, ApJ, 556, 872

Allen, P. R., Koerner, D. W., Reid, I. N., \& Trilling, D. E. 2005, ApJ, 625, 385

Baraffe, I., Chabrier, G., Barman, T. S., Allard, F., \& Hauschildt, P. H. 2003, A\&A, 402, 701
Barrado y Navascués, D., Stauffer, J. R., \& Jayawardhana, R. 2004, ApJ, 614, 386 Bodenheimer, P. 1965, ApJ, 142, 451

Bouy, H., Brandner, W., Martín, E. L., Delfosse, X., Allard, F., \& Basri, G. 2003, AJ, 126, 1526 
Burgasser, A. J., Kirkpatrick, J. D., Cruz, K. L., Reid, I. N., Leggett, S. K., Liebert, J., Burrows, A., \& Brown, M. E. 2006, ApJS, 166, 585

Burgasser, A. J., Kirkpatrick, J. D., \& Lowrance, P. J. 2005a, AJ, 129, 2849

Burgasser, A. J., Marley, M. S., Ackerman, A. S., Saumon, D., Lodders, K., Dahn, C. C., Harris, H. C., \& Kirkpatrick, J. D. 2002, ApJ, 571, L151

Burgasser, A. J., Reid, I. N., Leggett, S. K., Kirkpatrick, J. D., Liebert, J., \& Burrows, A. 2005b, ApJ, 634, L177

Burrows, A., Hubbard, W. B., Lunine, J. I., \& Liebert, J. 2001, Rev. Mod. Phys., 73, 719

Burrows, A., Sudarsky, D., \& Hubeny, I. 2006, ApJ, 640, 1063

Burrows, A., et al. 1997, ApJ, 491, 856

Chabrier, G., Baraffe, I., Allard, F., \& Hauschildt, P. 2000, ApJ, 542, 464

Chauvin, G., Lagrange, A.-M., Dumas, C., Zuckerman, B., Mouillet, D., Song, I., Beuzit, J.-L., \& Lowrance, P. 2005a, A\&A, 438, L25

Chauvin, G., et al. 2005b, A\&A, 438, L29

Close, L. M., et al. 2005, Nature, 433, 286

Cushing, M. C., Rayner, J. T., \& Vacca, W. D. 2005, ApJ, 623, 1115

Cushing, M. C., et al. 2006, ApJ, 648, 614

Dahn, C. C., et al. 2002, AJ, 124, 1170

Donahue, R. A. 1993, Ph.D. thesis, New Mexico State Univ.

Drilling, J. S., \& Landolt, A. U. 2000, in Allen's Astrophysical Quantities, ed. A. Cox (4th ed.; New York: Springer), 382

Duncan, D. K., \& Jones, B. F. 1983, ApJ, 271, 663

Duquennoy, A., \& Mayor, M. 1991, A\&A, 248, 485

Dyck, H. M., Benson, J. A., van Belle, G. T., \& Ridgway, S. T. 1996, AJ, 111, 1705

Eggen, O. J. 1964, AJ, 69, 570

Enoch, M. L., Brown, M. E., \& Burgasser, A. J. 2003, AJ, 126, 1006

Fleming, T. A., Molendi, S., Maccacaro, T., \& Wolter, A. 1995, ApJS, 99, 701

Geballe, T. R., et al. 2002, ApJ, 564, 466

Gizis, J. E., Reid, I. N., Knapp, G. R., Liebert, J., Kirkpatrick, J. D., Koerner, D. W., \& Burgasser, A. J. 2003, AJ, 125, 3302

Golimowski, D. A., et al. 2004, AJ, 127, 3516

Goto, M., et al. 2003, in Proc. SPIE, 4839, 1117

Gray, D. F. 1992, The Observation and Analysis of Stellar Photospheres (Cambridge: Cambridge Univ. Press)

Grenier, S., et al. 1999, A\&AS, 137, 451

Hartmann, L., Soderblom, D. R., Noyes, R. W., Burnham, N., \& Vaughan, A. H. 1984, ApJ, 276, 254

Hayward, T. L., Brandl, B., Pirger, B., Blacken, C., Gull, G. E., Schoenwald, J., \& Houck, J. R. 2001, PASP, 113, 105

Herbig, G. H. 1965, ApJ, 141, 588

Jaschek, M. 1978, Bull. Cent. Données Stellaires, 15, 121

Jones, B. F., Fischer, D., Shetrone, M., \& Soderblom, D. R. 1997, AJ, 114, 352

Kawaler, S. D. 1988, ApJ, 333, 236

Kirkpatrick, J. D. 2005, ARA\&A, 43, 195

Kirkpatrick, J. D., Barman, T. S., Burgasser, A. J., McGovern, M. R., McLean, I. S., Tinney, C. G., \& Lowrance, P. J. 2006, ApJ, 639, 1120

Kirkpatrick, J. D., Dahn, C. C., Monet, D. G., Reid, I. N., Gizis, J. E., Liebert, J., \& Burgasser, A. J. 2001, AJ, 121, 3235

Kirkpatrick, J. D., et al. 1999, ApJ, 519, 802

. 2000, AJ, 120, 447

Koen, C., \& Eyer, L. 2002, MNRAS, 331, 45

Kraft, R. P. 1970, in Spectroscopic Astrophysics. An Assessment of the Contributions of Otto Struve, ed. G. H. Herbig (Berkeley: Univ. California Press), 385

Krishnamurthi, A., et al. 1998, ApJ, 493, 914
Liu, M., et al. 2005, BAAS, 37, 1326

Liu, M. C., Leggett, S. K., Golimowski, D. A., Chiu, K., Fan, X., Geballe, T. R., Schneider, D. P., \& Brinkmann, J. 2006, ApJ, 647, 1393

Lowrance, P. J., et al. 2000, ApJ, 541, 390

Lucas, P. W., Roche, P. F., Allard, F., \& Hauschildt, P. H. 2001, MNRAS, 326, 695

Luhman, K. L., \& Potter, D. 2006, ApJ, 638, 887

Luhman, K. L., Stauffer, J. R., \& Mamajek, E. E. 2005, ApJ, 628, L69

McLean, I. S., McGovern, M. R., Burgasser, A. J., Kirkpatrick, J. D., Prato, L., \& Kim, S. S. 2003, ApJ, 596, 561

Mermilliod, J. C. 1981, A\&A, 97, 235

Metchev, S. A. 2006, Ph.D. thesis, Caltech

Metchev, S. A., \& Hillenbrand, L. A. 2004, ApJ, 617, 1330

Mohanty, S., Basri, G., Jayawardhana, R., Allard, F., Hauschildt, P., \& Ardila, D. 2004a, ApJ, 609, 854

Mohanty, S., Jayawardhana, R., \& Basri, G. 2004b, ApJ, 609, 885

Montes, D., López-Santiago, J., Gálvez, M. C., Fernández-Figueroa, M. J., De Castro, E., \& Cornide, M. 2001, MNRAS, 328, 45

Neuhäuser, R., Guenther, E. W., Wuchterl, G., Mugrauer, M., Bedalov, A., \& Hauschildt, P. H. 2005, A\&A, 435, L13

Nielsen, E. L., Close, L. M., Guirado, J. C., Biller, B. A., Lenzen, R., Brandner, W., Hartung, M., \& Lidman, C. 2005, Astron. Nachr., 326, 1033

Nordström, B., et al. 2004, A\&A, 418, 989

Noyes, R. W., Hartmann, L. W., Baliunas, S. L., Duncan, D. K., \& Vaughan, A. H. 1984, ApJ, 279, 763

Perryman, M. A. C., et al. 1997, A\&A, 323, L49

1998, A\&A, 331, 81

Pinsonneault, M. H., Kawaler, S. D., Sofia, S., \& Demarque, P. 1989, ApJ, 338, 424

Pinsonneault, M. H., Stauffer, J., Soderblom, D. R., King, J. R., \& Hanson, R. B. 1998, ApJ, 504, 170

Prosser, C. F. 1992, AJ, 103, 488

Radick, R. R., Skiff, B. A., \& Lockwood, G. W. 1990, ApJ, 353, 524

Randich, S. 2000, in ASP Conf. Ser. 198, Stellar Clusters and Associations:

Convection, Rotation, and Dynamos, ed. R. Pallavicini, G. Micela, \& S. Sciortino (San Francisco: ASP), 401

Soderblom, D. R., Oey, M. S., Johnson, D. R. H., \& Stone, R. P. S. 1990, AJ, 99, 595

Stassun, K. G., Mathieu, R. D., \& Valenti, J. A. 2006, Nature, 440, 311

Stauffer, J. R., Schultz, G., \& Kirkpatrick, J. D. 1998, ApJ, 499, L199

Stauffer, J. R., et al. 1999, ApJ, 527, 219

Stephens, D. C., \& Leggett, S. K. 2004, PASP, 116, 9

Strassmeier, K., Washuettl, A., Granzer, T., Scheck, M., \& Weber, M. 2000, A\&AS, 142, 275

Tinney, C. G., Butler, R. P., Marcy, G. W., Jones, H. R. A., Penny, A. J., McCarthy, C., Carter, B. D., \& Bond, J. 2003, ApJ, 587, 423

Troy, M., et al. 2000, in Proc. SPIE, 4007, 31

Tsuji, T. 2002, ApJ, 575, 264

Tsuji, T., \& Nakajima, T. 2003, ApJ, 585, L151

van Leeuwen, F., Alphenaar, P., \& Meys, J. J. M. 1987, A\&AS, 67, 483

Vilhu, O. 1984, A\&A, 133, 117

Voges, W., et al. 2000, IAU Circ., 7432, 1

Vrba, F. J., et al. 2004, AJ, 127, 2948

White, R. J., Gabor, J., \& Hillenbrand, L. A. 2006, AJ, submitted

Wilson, J. C., Kirkpatrick, J. D., Gizis, J. E., Skrutskie, M. F., Monet, D. G., \& Houck, J. R. 2001, AJ, 122, 1989

Wizinowich, P., et al. 2000, PASP, 112, 315

Wright, J. T., Marcy, G. W., Butler, R. P., \& Vogt, S. S. 2004, ApJS, 152, 261

Note added in proof.-A second unusually cool young brown dwarf near the L/T transition. HN Peg B, was subsequently reported by K. L. Luhman et al. (ApJ, in press [2006], astro-ph/0609464). These authors agree that the anomalous behavior of the 300 Myr old HN Peg B (T2.5) and HD 203030 B (L7.5) indicates that the temperature at the L/T transition is dependent on surface gravity. 